\title{
Intracranial dural AV fistula- An unfolding enigma
}

\author{
Rajesh Shenoy ${ }^{1}$, Ravi Ichalakaranji ${ }^{2, *}$, Prakash Mahantshetti ${ }^{3}$ \\ ${ }^{1}$ Professor, ${ }^{2}$ Mch Resident, ${ }^{3}$ Assistant Professor, KLE University, Belgaum, Karnataka, India
}

*Corresponding Author:

Email: ravi.ichalkaranji@gmail.com

\begin{abstract}
An Intracranial dural arteriovenous fistulas (DAVF), is an abnormal direct connection (fistula) between a meningeal artery and a meningeal vein or dural venous sinus $\& /$ cortical vein 1 . When there are multiple fistulas they are termed as dural arteriovenous malformations and comprise $10-15 \%$ of all intracranial arteriovenous malformations 1.

Here we present a case of $52 \mathrm{yr}$ old patient who presented to our casualty with history of sudden onset of headache, giddiness, and vomiting, followed by loss of consciousness. Neurological examination revealed impairment of consciousness, no verbal response, with left hemiparesis (E2V1M5). Bilaterally, pupils were $5 \mathrm{~mm}$ and sluggishly reacting to light. Vitals were stable with B.P. recording of 140/80 $\mathrm{mm}$ of hg, and a pulse rate of $80 / \mathrm{min}$. Pre contrast CT scan showed an intraparenchymal haemorrhage with perilesional oedema in the right frontal region.

CT angiography brain showed a intracranial dural AVF with varix and adjacent intraparenchymal haemorrhage.

D.S.A. revealed a Borden type III \& Cognard type IV - midline dural Arteriovenous fistula in the region of mid superior sagittal sinus and along the cerebral falx , with a large venous ectasia/varix along the anterior cerebral falx.

Management: Patient underwent a fronto parietal rectangular craniotomy Right posterior frontal, parasagittal engorged bunch of focal cortical veins was cauterized. Underlying haematoma was evacuated and the underlying venous ectasia/ varix identified. It was found to be thrombosed. A large draining vein was found which was clipped and any consequent brain swelling was looked for, before finally excising it in toto.

Post -op period was uneventful, with the patient gradually improving in sensorium with residual hemiplegia and aphasia.

Although recent developments in catheter intervention has made it the preferred option in most of the cases, surgery is still preferred in some select cases.
\end{abstract}

Keywords: Intracranial dural AV fistula, Superior sagittal sinus, Cognard and borden classification, CT andiography, Digital subtraction angiography.

\section{Introduction}

An intracranial dural arteriovenous fistulas (DAVF), is an abnormal direct connection (fistula) between a meningeal artery and a meningeal vein or dural venous sinus $\& /$ cortical vein. ${ }^{7}$ When there are multiple fistulas they are termed as dural arteriovenous malformations and comprise $10-15 \%$ of all intracranial arteriovenous malformations. ${ }^{7}$ Most of them are located in the posterior fossa or in the region of the cavernous sinus. Patients may be asymptomatic or symptoms may range from mild symptoms to fatal haemorrhage, depending on the location and venous drainage pattern, which ultimately dictates management strategy. ${ }^{3}$ Although recent developments in catheter intervention has made it the preferred option in most of the cases, surgery is still preferred in some select cases. ${ }^{7}$

\section{Case report}

A $52 \mathrm{yr}$ old patient presented to our institute with history of sudden onset of headache, giddiness, and vomiting, followed by loss of consciousness. Neurological examination revealed impairment of consciousness, no verbal response, with left hemiparesis (E2V1M5). Bilaterally, pupils were $5 \mathrm{~mm}$ and sluggishly reacting to light. Vitals were stable with B.P. recording of 140/80 $\mathrm{mm}$ of hg, and a pulse rate of $80 / \mathrm{min}$. Pre contrast computed tomography (CT) scan
Fig 1 showed an intraparenchymal haemorrhage with perilesional oedema in the right frontal region, centrum semi ovale and corona radiate measuring $5.2 \times 4.1 \times 4.5 \mathrm{cms}$ with extension into bilateral lateral ventricles and the $3^{\text {rd }}$ and 4thventricles. Diffuse subarachnoid hemorrhage noted in the bilateral frontoparietal regions, right sylvian cistern with mild generalized effacement of cortical sulci and basal cisterns suggestive of diffuse cerebral oedema. There is mass effect with midline shift to the left by $3.00 \mathrm{~mm}$.

Patient was intubated, anti-edema and all other supportive measures, instituted, and stabilized. CT angiography brain Fig 2 showed a dural avf with varix and adjacent intraparenchymal haemorrhage.

D.S.A Fig. 3 revealed a Borden type III \& Cognard type IV-midline dural Arteriovenous fistula in the region of mid superior sagittal sinus and along the cerebral falx , with a large venous ectasia/varix along the anterior cerebral falx. The dural AVF is supplied by arterial feeders from the right middle meningeal artery, transosseous branches of right superficial temporal artery with direct cortical venous drainage. The rest of the dural and cortical venous sinuses are normal.

Due to the concerns of raised I.C.P., due to the large size of the hematoma, easy accessibility, informed choice of the patient relatives, and cost effectiveness, surgery was decided as the treatment of choice. Patient underwent a fronto parietal rectangular craniotomy 
extending across the midline, exposing the superior sagittal sinus in the region of the dural avf, via a bicoronal skin incision behind the coronal suture.

Dura flap raised with a medial base towards the sinus, essentially cutting off the arterial meningeal feeders. Right posterior frontal, parasagittal engorged bunch of focal cortical veins was cauterized. (Fig. 4)

Underlying haematoma was evacuated and the underlying venous ectasia/varix identified. It was found to be thrombosed. (Fig. 5)

A large draining vein was found which was clipped Fig. 6 and any consequent brain swelling was looked for, before finally excising it in toto.

Duroplasty with fascia and wound closed in layers over a sub galeal drain. Post -op period was uneventful, with the patient gradually improving in sensorium with residual hemiplegia and aphasia.
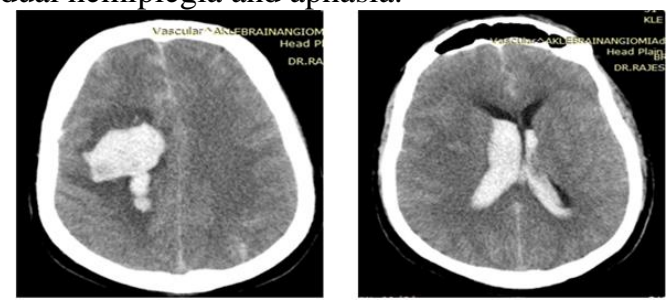

Fig. 1: Showing right post frontal intraparenchymal hemorrhage with extension into lateral, $3^{\text {rd }}$ and $4^{\text {th }}$ ventricle
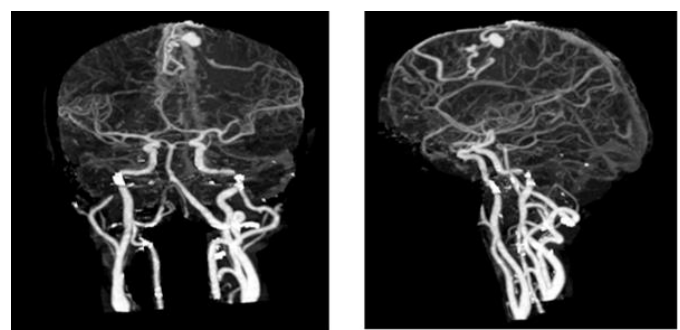

Fig 2: Angio brain showing dural AVF with adjacent varix

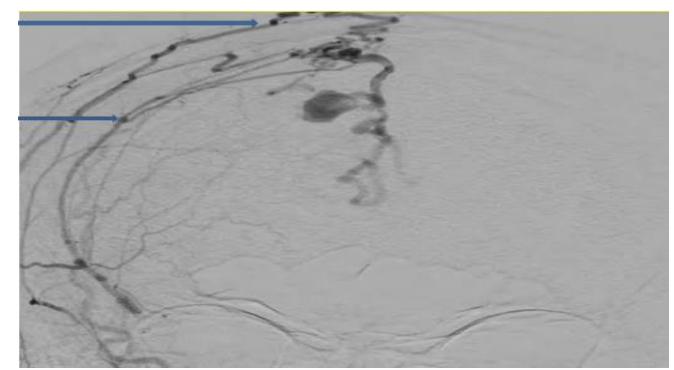

Fig. 3: DSA showing feeders from middle meningeal and superficial temporal artery with venous

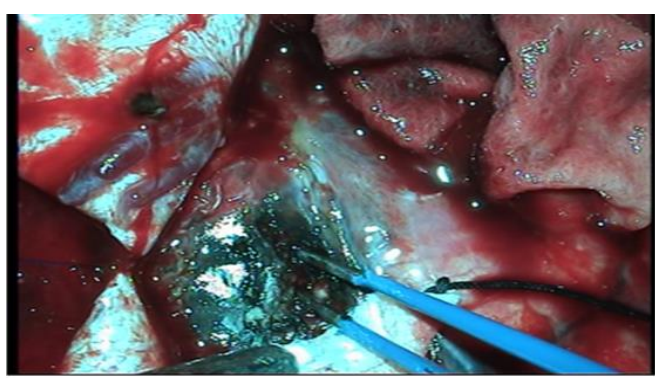

Fig. 4: Intraop image of dural vessels and focal engorged cortical veins

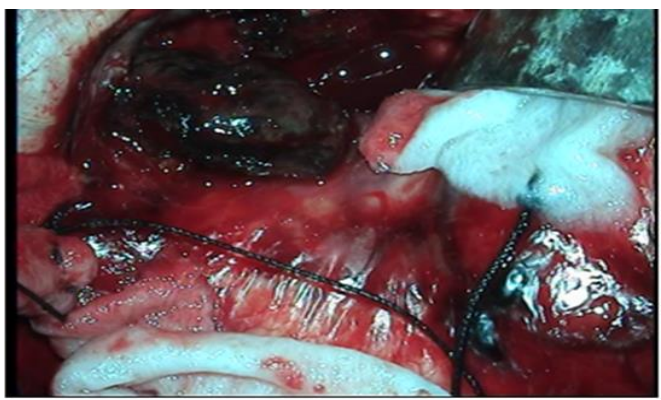

Fig. 5: Venous varix and hematoma

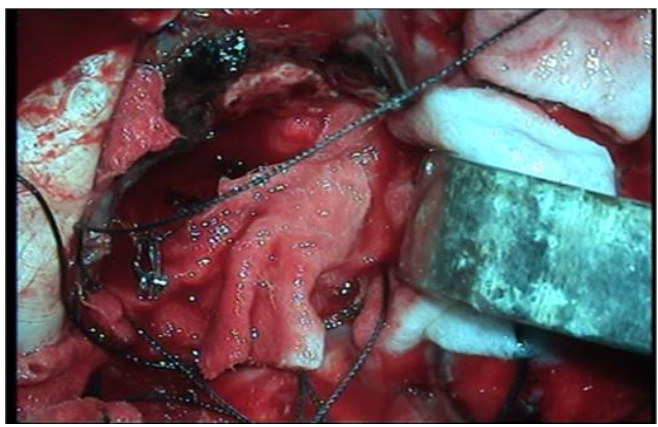

Fig. 6: Clipped venous varix
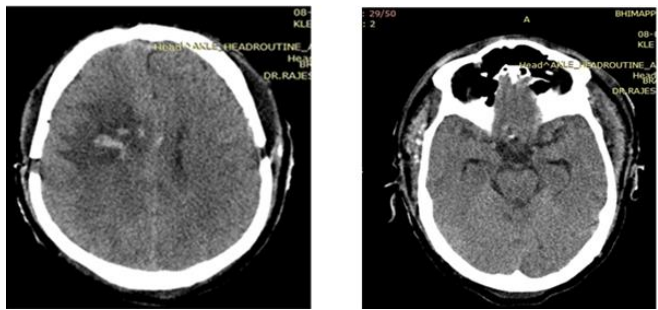

Fig. 7: Postoperative CT brain showing resolution of hematoma

\section{Discussion}

Dural arteriovenous fistulas are anomalous arteriovenous communications developed within a venous space contained between the two layers of the duramater and typically supplied by multiple feeders from arteries that supply the relevant part of the meninges and regional scalp vessels which give transosseous branches. ${ }^{8}$ They account for $10 \%-15 \%$ of all intracranial vascular malformations. ${ }^{9}$

supratentorial and lateral: (external carotid artery ${ }^{2}$ Middle Meningeal Artery 
Superficial Temporal Artery (transosseous branches) Anterior cranial fossa: (internal carotid artery)

Ethmoidal branches of the ophthalmic artery

Cavernous sinus: (internal and external carotid arteries)

Meningohypophyseal Trunk and inferolateral trunk

Accessory Meningeal Artery

Posterior cranial fossa: (vertebral and external carotid arteries)

Vertebral Arteries (both dural and muscular branches)

Occipital and ascending pharyngeal arteries

\section{Location $^{2}$}

Transverse/sigmoid sinus

Most common

Least likely to have retrograde venous drainage

Cavernous Sinus (indirect caroticocavernous fistula)

Superior Sagittal Sinus

Straight Sinus

Other venous sinuses

Anterior Cranial Fossa

Typically only ICA supply due to meningeal supply of this region

Frequently associated with retrograde venous drainage Tentorium: frequently associated with retrograde venous drainage.
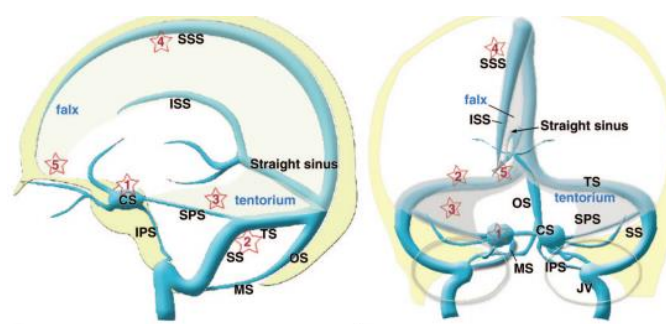

Fig. 1: Drawings $(a \stackrel{\text { b. }}{=}$ lateral view, b. $=$ anteroposterior view) illustrate the most common location of dural AVFs: 1= cavernous sinus (CS) $(20 \%-40 \%$ of cases), $2=$ transverse-sigmoid sinus (TS, SS) $(20 \%-60 \%), 3=$ tentorium $(12 \%-14 \%), 4=$ superior sagittal sinus (SSS) $(8 \%)$, and $5=$ anterior fossa $(2 \%-3 \%)$, IPS = inferior petrosal sinus, ISS = inferior sagittal sinus, $\mathbf{J V}=$ jugular vein, $\mathrm{MS}=$ marginal sinus, $\mathrm{OS}=$ occipital sinus, $\mathrm{SPS}=$ superior petrosal sinus

Dural arteriovenous fistulas are usually acquired lesions.1 Inciting events preceding the development of DAVF have included trauma, ${ }^{2-4}$ cranial surgery,,${ }^{3,4}$ otitis or sinus infection, ${ }^{5}$ hormonal changes (e.g., pregnancy, estrogen replacement), and an association with meningiomas. ${ }^{6}$

Davfs are caused by angiogenic factors released from organizing thrombus leading to invasion of small dural arteries and formation of small arteriovenous shunts. ${ }^{7}$

Venous hypertension may also increase angiogenic activity or lead to local tissue hypoxia that initiates neoangiogenesis and endothelial proliferation, leading to Davf formation. ${ }^{7}$

Another theory suggests that DAVFs arise from naturally occurring dormant channels between dural arteries and sinuses, which opens when the sinus is occluded and venous pressure is increased. ${ }^{7}$

In immune histochemical studies, expression of basic fibroblast growth factor (bFGF) and vascular endothelial growth factor (VEGF) has been identified in the wall of the dural sinuses in patients with davfs. ${ }^{7}$

Patient symptoms may be characterized as either non aggressive (benign) (eg., tinnitus) or aggressive (eg., intracranial haemorrhage)Table $1 .^{9}$

Table 1: Symptoms of intracranial dural

\begin{tabular}{|l|c|c|c|c|c|}
\hline AVFs Symptoms & $\begin{array}{c}\text { Cavernous } \\
\text { sinus (\%) }\end{array}$ & $\begin{array}{c}\text { Transverse- } \\
\text { sigmoid } \\
\text { sinus (\%) }\end{array}$ & $\begin{array}{c}\text { Tentoriu } \\
\mathbf{( \% )}\end{array}$ & $\begin{array}{c}\text { Superior } \\
\text { sagittal } \\
\text { sinus(\%) }\end{array}$ & $\begin{array}{c}\text { Anterior } \\
\text { fossa } \\
(\%)\end{array}$ \\
\hline Ocular symptoms & $80-97$ & ----- & ------ & ----- & ------ \\
\hline Cranial nerve deficits & $44-77$ & $7-12$ & $14-17$ & ----- & ------ \\
\hline Bruit, tinnitus & $40-50$ & $40-42$ & $70-88$ & ------ & ------ \\
\hline Headache & ----- & $46-76$ & $8-24$ & 50 & $12-15$ \\
\hline Visual symptoms & $28-38$ & $12-28$ & ----- & ----- & ----- \\
\hline Central nerve deficits & 3 & $10-20$ & $23-42$ & 29 & $5-33$ \\
\hline $\begin{array}{l}\text { Intracranial } \\
\text { hemorrhage }\end{array}$ & Rare & $15-28$ & $60-74$ & 23 & $44-84$ \\
\hline dementia & ----- & Rare & ---- & 5 & ---- \\
\hline
\end{tabular}

Although there have been many classification schemes suggested for DAVF, the classification proposed by borden and cognard are the most widely used. $^{7}$

The Borden classification system categorizes DAVF based on the site of venous drainage (dural sinus versus cortical vein) and the absence or the presence of cortical venous drainage. ${ }^{10}$

The cognard system not only notes these features but also takes into account the direction of flow in an involved dural sinus (antegrade versus retrograde), as 
well as the absence or presence of venous ectasia(s) in recruited cortical veins. ${ }^{10}$

1. Borden classification

Type 1: venous drainage into a dural sinus with no CVD

Type 2: Venous drainage into a dural sinus with associated CVD

Type 3: Drainage into cortical veins (CVD)

2. Cognard classification

Type I: Venous drainage into dural sinus with antegrade flow

Type IIa: Venous drainage into dural sinus with retrograde flow

Type IIb: Venous drainage into dural sinus with antegrade flow and CVD

Type II $(a+b)$ : Venous drainage into dural sinus with retrograde flow \& CVD

Type III: Venous drainage into cortical veins (CVD)

Type IV: CVD with associated venous ectasia(s)

Type V: Venous drainage into spinal Perimedullary veins.

Symptoms associated with dural avf can be due to either increased dural sinus drainage or the development of cortical venous hypertension. ${ }^{12}$ Anterior fossa lesions are typically supplied by ethmoidal arteries and drain into the cavernous sinus, these davfs typically present with ocular symptoms including proptosis, chemosis, ophthalmoplegia, decreased visual acuity, or retro orbital pain because of their proximity to the orbit.

Middle fossa lesions commonly drain into the transvers or sigmoid sinus These fistulas typically cause symptoms of pulsatile tinnitus because of the close proximity to the auditory apparatus. ${ }^{12}$ Fistulas that drain into the superior sagittal sinus or deep venous system produce symptoms of global venous congestion and raised ICP, and may manifest with symptoms of hydrocephalus, papilloedema, seizures, or dementia.

Brainstem davfs, though less common than other locations, can present with cranial neuropathies and /or quadriparesis. $^{12}$ The presence of cortical venous hypertension in addition, typically causes more severe symptoms, including Intracranial haemorrhage and neurological deficits which include progressive dementia, seizures, parkinsonism, and other focal neurological deficits including aphasia, alexia, weakness, paraesthesias, and ataxia. ${ }^{12}$

Non contrast computed tomography (CT) and conventional magnetic resonance (MR) imaging often seem unremarkable with benign DAVF. However, these studies may demonstrate the complications of aggressive lesions with CVD, including hemorrhage, venous congestion with edema, venous aneurysms, tortuous cortical veins in a pseudophlebitic pattern, and parenchymal or leptomeningeal enhancement. ${ }^{10}$

CT and MR angiography (CTA and MRA, respectively) may be used to screen patients suspected with DAVF, can grade or classify these lesions when detected, as well as evaluate for response to treatment. ${ }^{10}$

Both CTA and MRA may visualize the fistula itself as prominent vessels associated with the meninges or dural sinus wall, as well as detect enlarged feeding arteries, early dural sinus opacification, and prominent draining veins. ${ }^{10}$

Despite these advances in MR and CT imaging, catheter angiography remains the definitive imaging study and the gold standard for evaluation of DAVF because of its superior spatial and temporal resolution. It can delineate both the arterial supply and the venous drainage of the fistula, as well as identify high-risk features including CVD, venous outflow obstruction, and arterial pedicle or venous aneurysms. Catheter angiography is also excellent for evaluation of any associated dural venous sinus thrombosis or occlusion. Finally it provides accurate information for planning endovascular or surgical treatment. ${ }^{10}$

\section{General treatment approaches}

Management of DAVF should be based on patient characteristics, symptom severity, and risk of serious sequelae, the latter being primary determined by the presence or absence of cortical venous reflux. ${ }^{10} \mathrm{DAVF}$ without high-risk features may be managed conservatively with an acceptably low rate of serious complications. In these instances, treatment should be tailored to palliation of intolerable symptoms. ${ }^{10}$

Spontaneous regression of dural AVFs has been reported. However, patients with benign DAVF electing conservative management should undergo clinical and imaging follow-up given the small risk of conversion to an aggressive lesion. ${ }^{10}$

Endovascular Interventions include Transarterial embolization (TAE) with particles, Transvenous Coil embolization, TAE with n-butyl -2-cyanoacrylate and Stent Placement. ${ }^{9}$

Stereotactic radiosurgery has the advantage of decreased invasiveness and fewer short-term complications, whereas the disadvantage is the delayed response (approximately 6-12 months) after irradiation. ${ }^{9}$ Results from large centers have been mixed, with cure rates ranging from $50 \%$ to $93 \%$ for DAVF patients treated with SRS. ${ }^{13,17}$ The majority of studies used a median dose of approximately $20 \mathrm{~Gy}$, with a range based on the location and whether adjunctive treatment with embolization or surgery was employed. In published studies, there is a trend toward a higher rate of success depending on location, with cavernous sinus lesions having the highest rate of successful obliteration. ${ }^{17}$

Surgical treatment methods for DAVF include surgical excision of involved meningeal arteries and veins, packing of the diseased dural sinus, as well as skeletonization of the involved dural sinus with disconnection of draining leptomeningeal veins. ${ }^{10}$ Risks of surgical repair of DAVF include blood loss, 
intracranial hemorrhage, arterial infarct, venous infarct, and cerebrospinal fluid leakage. ${ }^{10}$

\section{Conclusion}

Davfs are venous-based rather than arterial-based lesions and treatment should focus on the venous side of the fistula. ${ }^{7}$ Microsurgical repair is a safe and effective treatment method for DAVF, either alone or in combination with endovascular embolization. ${ }^{10}$

\section{References}

1. Kelly D. Flemming, Robert D. Brown Jr. Epidemiology and Natural History of Intracranial Vascular Malformations.In: Winn, H. Richard editors Youmans \& Winn Neurological Surgery. Seventh edition. Philadelphia: Elsevier 2017.

2. J Dennery, B Ignacio: Post-traumatic arteriovenous fistula between the external carotid arteries and the superior longitudinal sinus: report of a case. Can J Surg 1967;10:333-336.

3. M Nabors, C Azzam, F Albanna, et al.: Delayed postoperative dural arteriovenous malformations. Report of two cases. J Neurosurg 1987;66:768-772.

4. A Watanabe, Y Takahara, Y Ibuchi, et al.: Two cases of dural arteriovenous malformation occurring after intracranial surgery. Neuroradiol 1984;26:375-380.

5. N Little, D Piepgras: Intracranial dural arteriovenous fistula. H Batjer Cerebrovascular Disease. 1997 Lippincott-Raven Philadelphia 799-809.

6. J Ugrinovski, M Vrcakovski, K Lozance: Dural arteriovenous malformation secondary to meningioma removal. Br J Neurosurg 1989;3:603-07.

7. Intracranial dural arteriovenous fistula- Anna Piippo.

8. Bruno Di Muzio, Frank Gaillard et al. Dural arteriovenous fistula. Radiopedia.

9. Hiro Kiyosue, MD, Yuzo Hori, MD, Mika Okahara, MD. Treatment of Intracranial Dural Arteriovenous Fistulas: Current Strategies Based on Location and Hemodynamics, and Alternative Techniques of Transcatheter Embolization. Radiographics 2004;24:1637-53.

10. Timothy R. Miller, MD; Dheeraj Gandhi, MD et al. Intracranial Dural Arteriovenous Fistulae Clinical Presentation and Management Strategies. Stroke 2015;46:2017-25.

11. Gandhi D, Chen J, Pearl M et al. Intracranial dural arteriovenous fistulas: classification, imaging findings, and treatment. AJNR Am J Neuroradiol 2012;33(6):100713.

12. Cranial arteriovenous malformations and cranial dural arteriovenous fistulas Neurosurgery clinics of North America January 2012;23(1).

13. 13BE Pollock, et al.: Stereotactic radiosurgery and particulate embolization for cavernous sinus dural arteriovenous fistulae. Neurosurg 1999;45:459-66.

14. CP Cifarelli, et al.: Gamma knife radiosurgery for dural arteriovenous fistulas. Neurosurg 2010;67:1230-35.

15. M Soderman, et al.: Gamma knife surgery for dural arteriovenous shunts: 25 years of experience. $J$ Neurosurg 2006;104:867-75.

16. Yang HC, et al.: Stereotactic radiosurgery with or without embolization for intracranial dural arteriovenous fistulas. Neurosurg 2010;67:1276-83.

17. Wu HM, et al.: Gamma Knife surgery for the management of intracranial dural arteriovenous fistulas. $J$ Neurosurg 2006;105(suppl):43-51. 